\title{
AVALIAÇÃO DAS AÇÕES DE CONTROLE DA INFECÇÃO ESQUISTOSSOMÓTICA NAS LOCALIDADES DE CACHOEIRA - BAHIA, BACIA DO PARAGUAÇU, 1982-1992
}

\author{
Vilma Sousa Santana, Maria da Glória Teixeira e Cristina C.P. Santos
}

\begin{abstract}
Esta investigação busca avaliar a efetividade das ações destinadas ao Programa de Controle da Esquistossomose, PCE, em um municipio da Bahia, a Região da Bacia do Paraguaçu. Os dados são provenientes das fichas PCE-111 da SUCAM/FNS, registrados rotineiramente, nas 111 localidades identificadas entre 1982 e 1992 . A infecçãofoi diagnosticada através de exames de fezes (Katz-Kato). A análise da prevalência da infecção baseou-se nas tendências da evoluçẫo temporal e distribuição espacial. Identificou-se quatro padrões típicos de evolução, sendo que os mais comuns são os de recorrência da infecção $(67,6 \%)$. O controle ocorreu em 22,5\% enquanto a expansão da infeç̧ão apareceu em apenas $9,9 \%$ das localidades. No geral, a tendência foi de redução da prevalência, particularmente no período entre 1982 e 1985, ascendendo posteriornente para valores, que não atingem o patamar inicial. Verificou-se também impacto na redução relativa do número de localidades que apresentavam prevalência abaixo de 5\%, indicativas de controle. Não foi possivel examinar a evolução da intensidade da infecção ou mesmo da ocorrência de formas graves ou óbitos que poderiam apresentar um quadro mais completo da efetividade das açôes desse programa.
\end{abstract}

Palavras-chaves: Esquistossomose. Controle. Avaliação. Tendênciatemporal. Distribuição espacial.

A avaliação da efetividade de programas de saúde pública vem gradualmente sendo considerada como procedimento imperativo no âmbito das políticas públicas (OMS, 1981) 23. A sua realização, entretanto, se depara com limitações decorrentes da própria natureza do objeto saúde e de suas intrincadas relações com aspectos econômicos, sociais e culturais, tomando-se ainda mais complexa, quando se trata de doenças crônicas que necessitam de apoio médico e laboratorial para serem identificadas ${ }^{13}$. A urgência dessas avaliações se impõe face à necessidade de: a) otimizar o uso dos recursos disponíveis; b) fundamentar a tomada de decisões frente ao avanço científico eà diversidade tecnológica do mercado ou à própria efetividade das ações; c) informar a população sobre o impacto epidemiológico; d) realizar projeções do alcance de metas epidemiológicas, estabelecidas no tempo

Instituto de Saúde Coletiva, Universidade Federal da Bahia, Salvador, BA.

Financiamento: Fundação Nacional de Saúde/Ministério da Saúde.

Endereço para correspondência: Dra. Vilma Sousa Santana. ISC/UFBA. R. Pe. Feijó 29, $4^{\circ}$ andar, Canela, 40140-170 Salvador, BA.

Recebido para publicação em 08/01/96. (controle, eliminação, erradicação) $^{30}$.

Após o conhecimento, em 1953, da grande extensão geográfica de áreas de ocorrência da esquistossomosse no Brasil, evidência da sua importância como problema de saúde pública ${ }^{25}$, o Governo Federal criou a Campanha Contra a Esquistossomose, que esteve sob a responsabilidade gerencial de diferentes instituições de saúde. Suas atividades, inicialmente, foram bastante limitadas em virtude do estado do conhecimento, àquela época, sobre a história natural da doença e instrumentos de controle adequados. Em 1976, o Programa Especial de Controle da Esquistossomose, PECE ${ }^{10}$ surgiu sob a responsabilidade da Superintendência de Campanhas (SUCAM) contando com recursos orçamentários e programáticos específicos. Esta iniciativa deveuse, principalmente, ao aparecimento de uma droga, o oxamniquine, reconhecida como de grande eficácia e de poucos efeitos colaterais graves, administrável em dose única, o que viabilizava a sua utilização em massa $^{31}$. Dados de estudos com esta e outras drogas, como o praziquantel, apontaram para o efeito do tratamento na prevenção da evolução das formas graves da doença e da possibilidade de diminuir a 
Santana VS, Teixeira MG, Santos CCP. Avaliaçâo das ações de controle da infecção esquistossomótica nas localidades de Cachoeira - Bahia, Bacia do Paraguaçu, 1982-1992. Revista da Sociedade Brasileira de Medicina Tropical 29:185-195, mar-abr, 1996.

prevalência da infecção ${ }^{71528}$, o que fortaleceu ainda mais, a decisão de aplicação de quimioterapia em larga escala. Vale ressaltar que o PECE foi alicerçado não só no uso do oxamniquine em massa, mas previa também atividades de controle de moluscos, de educação em saúde e melhorias sanitárias em áreas localizadas ${ }^{101831}$.

Centrado nessas ações, o PECE foi desenvolvido até 1980, quando de programa especial da SUCAM passa a Programa de Controle da Esquistossomose (PCE), mantendo-se o mesmo escopo de atividades. O PCE teve a revisão de suas estratégias iniciada em 1986 , mas somente em 1989 , novas normas ${ }^{12}$ foram elaboradas visando a obtenção de uma melhor racionalidade, conseqüência da retração de recursos, e também da necessidade de padronização da metodologia que permitisse a comparabilidade dos resultados alcançados ${ }^{31}$. O PCE sofreu alterações metodológicas ao longo do tempo, como também descontintuidades decorrentes de mudanças administrativas. Ademais, de início, era uma proposta centralizada e vertical, e mais recentemente passa para uma atuação mais descentralizada por força da implantação do Sistema Único de Saúde, sem deixar de desenvolver as atividades programadas pelo nível central do sistema.

A avaliação do PCE vem sendo realizada, principalmente, com dados coletados rotineiramente nos serviços de saúde da SUCAM/FNS ${ }^{2}{ }^{14}$. A maioria das avaliações das ações de controle da esquistossomose enfoca o efeito da quimioterapia. Isto se deve, provavelmente, à complexidade, especificidade e ainda pouca familiaridade dos profissionais de saúde com abordagens empíricas de aspectos cognitivos, comportamentais, sejam individuais ou coletivos, que se entrelaçam estreitamente com a conformação cultural dos grupos humanos.

Em 1975, Barbosa ${ }^{3}$ identificou uma redução na prevalência e intensidade da infeç̧ão em algumas pequenas localidades do Estado de Pernambuco, tanto naquelas submetidas à quimioterapia quanto naquelas não tratadas, levando à hipótese de ser este efeito decorrente de intervenções educativas e de saneamento. Em um município de Minas Gerais,
Coura e $\operatorname{cols}^{16}$ registraram uma redução da prevalência da infecção de $60,8 \%$ para $36,2 \%$ em um intervalo de tempo de 10 anos, "sem nenhum tipo de intervenção". Achados semelhantes foram reportados em áreas da Índia e Etiópia ${ }^{20}{ }^{23}$, que foram atribuídos a fenômenos climáticos ocorridos à epoca.

A efetividade da quimioterapia sobre a prevalência da infecção esquistossomótica vem sendo questionada em relação à permanência dos seus efeitos, desde que vem sendo notado que prevalências de infecção retornam aos niveis anteriores em um certo periodo de tempo. Sleigh e cols $\mathrm{s}^{32}$ em 1981, estimaram este intervalo em três anos, enquanto Santos \& Coura $^{27}$ em um período de seis anos. Contudo, os achados relatados por Bina ${ }^{7}$ e Bina \& Prata $^{8}$, indicando que o tratamento medicamentoso específico reduzia a incidência de formas graves, e também melhorava a evolução clínica dessas formas graves quando já instaladas, notadamente pela involução da hepatoesplenomegalia, mesmo que os pacientes continuassem residindo em área endêmica provocaram uma reviravolta nas opiniões sobre a importância e benefício desse tratamento. Assim, não somente poder-se-ia esperar que o tratamento em massa reduzisse a prevalência da infecção, mas a sua aplicação seria de grande valor nos programas de prevenção, porquanto promoveria o controle das formas graves, ainda que não seja suficiente para se atingir o controle da infecção.

Embora representem uma contribuição valiosa para a tomada de decisão de administradores de saúde, as avaliações do PCE ressentem-se por serem, em sua maioria, estudos realizados com dados de rotina dos serviços de saúde, cujas conclusões podem estar distorcidas devido à inadequação da coleta, armazenagem e processamento dos dados, além de constantes descontinuidades neste processo. Além disso, esses estudos têm utilizado como unidade de observação, o município ou estado, considerado como uma base de informação muito heterogênea para avaliação de uma doença de distribuição epidemiológica predominantemente focal.

O propósito deste trabalho é contribuir para a 
Santana VS, Teixeira MG, Santos CCP. Avaliação das ações de controle da infecção esquistossomótica nas localidades de Cachoeira-Bahia, Bacia do Paraguaçu, 1982-1992. Revista da Sociedade Brasileira de Medicina Tropical 29:185-195, mar-abr, 1996.

melhoria dos Programas de Controle da Esquistossomose no Brasil, produzindo conhecimento através da avaliação do impacto epidemiológico do conjunto de atividades desenvolvidas no âmbito do município estudado, que implicaram em alterações do perfil epidemiológico dessa endemia. Vale ressaltar, que năo se trata de uma avaliação específica do PCE. De fato, adota-se o modelo black box. que toma as várias ações potencialmente importantes na produção do efeito, em seu conjunto ${ }^{22}$. Portanto, aqui não são analisadas apenas as ações desenvolvidas pela FNS, certamente as mais proeminentes no Estado da Bahia neste aspecto, mas também atividades deenvolvidas como parte dos Programas de Apoio ao Pequeno Produtor Rural (PDRI-Paraguaçu), as Secretarias Estadual e Municipal de Saúde, organizações ou iniciativas filantrópicas, entre outras. O objetivo desse estudo é o de avaliar a efetividade do programa de controle da esquistossomose desenvolvido pela FNS/SUCAM no período de 1982 a 1992, em um município da Bahia, Cachoeira. Para tanto, estudou-se a evolução temporal e distribuição espacial da prevalência da esquistossomose em localidades de um município considerado como área endêmica do Estado da Bahia.

\section{MATERIAL E MÉTODOS}

Este é um estudo de base agregada, ou ecológico, desenvolvido com dados relativos ao período de 1982 a 1992, provenientes do sistema de informações de endemias da SUCAM, hoje incorporada à Fundação Nacional de Saúde. Os dados disponíveis, do tipo secundário, coletados como procedimento rotineiro, possibilitaram a análise de tendência histórica como também da distribuição espacial de prevalências de infecção esquistossomótica em localidades de um município do Estado da Bahia, Região da Bacia do Paraguaçu.

\section{Descrição da área}

O município de Cachoeira é o mais populoso dessa Região, considerada como endêmica para a esquistossomose desde o estudo de Pellon realizado em 1950, quando todas as localidades da área foram pesquisadas. Cachoeira tem uma área de $403 \mathrm{~km}^{2}$. De acordo com o Censo de 1991, sua população era de 28.258 habitantes, sendo 14.172 residentes na área urbana e 14.086 na área rural, o que corresponde a uma densidade demográfica de $70,11 \mathrm{hab} / \mathrm{km}^{2}$. Suas principais atividades econômicas são a lavoura e a pecuária. Baseando-se nos dados provenientes do Reconhecimento Geográfico (RG), realizado pela FNS, Cachoeira possui 115 localidades, sendo que dentre as residências identificadas, cerca de $35 \%$ não dispõe de água tratada, que se concentram na sede do município. O sistema de esgotamento sanitárió praticamente inexistente, mesmo na área urbana. Quanto ao nível de educação, cerca de $53 \%$ da população acima de cinco anos não têm instrução ou possuem menos de um ano de escolaridade.

Os principais rios que banham o município de Cachoeira são o Paraguaçu, Pitanga e Lagoa Encantada. Essa bacia possui muitos córregos, formando criadouros ideais para os caramujos hospedeiros intermediários do Schistosomamansoni, sendo a espécie predominante a Biomphalaria glabrata. A Barragem de Pedra do Cavalo, que teve o seu lago formado em 1986, foi construída no leito do rio Paraguaçu para a produção de energia elétrica, o que ocasionou alagamento de algumas localidades ribeirinhas e deslocamento populacional para outras áreas. A mortalidade proporcional por doenças infecciosas e parasitárias, assim como a mortalidade infantil vêm decrescendo ao longo dos anos, seguindo a mesma tendência do Estado e também do país ${ }^{4}$.

\section{Coleta de dados}

Os dados utilizados neste estudo são oriundos dos trabalhos desenvolvidos pelo Programa de Controle da Esquistossomose ${ }^{11}$ no período de 1982 a 1992, coletados de acordo com as normas técnicas desse programa: as equipes de campo se dirigem à região e identificam cada um dos agregados populacionais existentes, qualquer que seja a dimensão. Em áreas consideradas endêmicas, procede-se de acordo com as seguintes etapas: 1) contato com a população para apresentação do trabalho que será realizado na localidade; 2) mapeamento da localidade, com a realização de 
Santana VS, Teixeira MG, Santos CCP. Avaliação das ações de controle da infecção esquistossomótica nas localidades de Cachoeira - Bahia, Bacia do Paraguaçu. 1982-1992. Revista da Sociedade Brasileira de Medicina Tropical 29:185-195, mar-abr, 1996.

croquis e identificação de todas habitações; 3) censo dos habitantes da localidade; 4) coleta de amostras fecais de cada um dos habitantes para realização de exames parasitológicos de acordo com o método Katz-Kato; 5) os resultados dos exames são comunicados a cada indivíduo, e caso seja positivo, fornece-se a medicação específica (oxamniquine em dose única de $15 \mathrm{mg} / \mathrm{kg}$ para adultos e $20 \mathrm{mg} / \mathrm{kg}$ para crianças até 15 anos de idade), que é tomada às vistas do guarda sanitário.

\section{Fonte de dados - a ficha PCE-11i}

Os dados analisados nessa investigação provêm da ficha resumo PCE-111 que é preenchida a nível local pelo inspetor sanitário. Estas fichas compõem o relatório histórico das açôes desenvolvidas em cada uma das iocalidades "trabalhadas" pelas equipes de trabalho de campo e ficam armazenadas nas sedes dos distritos. Identificou-se dados sobre inquéritos de 1982 a 1992. Estas fichas contêm, além de dados de identificação do município e da localidade, informações sobre a população, número de casas, número de exames de fezes realizados, número de exames com resul tados positivos para a presença de ovos de schistossoma, proporção de casos positivos, natureza da etapa de trabalho (levantamento, censo, avaliação, tratamento ou retratamento), número de pessoas tratadas, número de pessoas não tratadas e total de consumo de oxamniquine.

Em 1986, em virtude de dificuldades operacionais, os exames parasitológicos de fezes foram realizados em apenas uma faixa etária, 7 a 14 anos, o que limita a comparação dos resultados destes anos com os demais. Em outras situações, algumas localidades foram alvo de mais de um inquérito no ano, considerando-se, nesse caso, a média aritmética simples desses valores.

\section{Análise dos dados}

A base de dados foi criada no EPI-INFO5, e a análise desenvolvida com o SAS-PC, 6.0426. Controle de qualidade da digitação foi feita manualmente, revisando-se uma vez todas as listagens, quando então corrigiram-se os erros identificados.
Para cada localidade, foram construídos gráficos mostrando a prevalência da esquistossomose específica para cada ano do período em estudo. Em seguida, cada gráfico foi analisado, separadamente, descrevendo-se a curva de tendência temporal em um croquis feito à mão. À medida que certos tipos de distribuição se repetiram, identificou-se aquilo que se denominou - Padrões Típicos de Evolução. Localidades foram então classificadas em cada um desses padrões-típicos, de acordo com a sua própria distribuição gráfica da evolução da prevalência. Feito isso, procedeu-se a análise tabular para obtenção de dados descritivos das localidades, de acordo com os seus padrões típicos de evolução. Esses padrões foram localizados no mapa do município de acordo com os agregados populacionais a que pertenciam. Por fim, gráficos box plot para os valores da prevalência das localidades em cada ano foram elaborados, com o sentido de se descrever as distribuições de acordo com os percentis, média e mediana, o que permite uma visualização do impacto numa perspectiva relativa ao número de localidades.

\section{RESULTADOS}

No município de Cachoeira, identificou-se 115 localidades. Em 111 delas, dispunha-se de dados provenientes de inquéritos realizados pela FNS SUCAM. Não foram encontrados registros de inquéritos para os anos de 1989, 1990 e 1991. No ano de 1983, encontrou-se dados sobre inquérito' tratamento em apenas sete localidades. Ao contrário, em 1985, verificou-se que foram realizados mais de dois inquéritos/tratamentos em 79 localidades, o que levou a um total de 187 observações em um total de 108 localidades para aquele ano. Nos demais anos, observa-se um percentual de perdas de informações que variou entre 2,7 a $16,2 \%$ das localidades sob investigação (Tabela 1).

Para o conjunto de localidades desse município, elaborou-se um gráfico (Figura 1) que mostra a evolução temporal da proporção de localidades com prevalência acima de $15 \%$, valor considerado como elevado para esta Região. Identifica-se uma queda abrupta no primeiro ano após o início do estudo, que deve ser relativizada devido ao pequeno número (7) de localidades estudadas em 1983. A 
Santana VS. Teixeira MG, Santos CCP. Avaliação das ações de controle da infecção esquistossomótica nas localidades de Cachoeira - Bahia, Bacia do Paraguaçu, 1982-1992. Revista da Sociedade Brasileira de Medicina Tropical 29:185-195, mar-abr, 1996.

'Tabela 1 - Distribuição da prevalência de esquistossomose nas localidades do municipio de Cachoeira, no periodo entre 1982 a 1992.

\begin{tabular}{|c|c|c|c|c|c|c|c|}
\hline \multirow{3}{*}{ Ano } & \multirow{3}{*}{$\mathrm{N}$} & \multicolumn{6}{|c|}{ Prevalência } \\
\hline & & \multicolumn{2}{|c|}{$(0-5 \%)$} & \multicolumn{2}{|c|}{$(6-15 \%)$} & \multicolumn{2}{|c|}{$(>5 \%)$} \\
\hline & & $\mathrm{N}$ & $\%$ & $\mathrm{~N}$ & $\%$ & $\mathrm{~N}$ & $\%$ \\
\hline 1992 & 111 & 40 & 36 & 40 & 36 & 31 & 28 \\
\hline 1983 & 7 & 4 & 57 & 2 & 29 & 1 & 14 \\
\hline 1984 & 97 & 39 & 41 & 36 & 37 & 22 & 23 \\
\hline 1985 & 108 & 64 & 59 & 36 & 33 & 8 & 8 \\
\hline 1986 & 108 & 68 & 63 & 25 & 23 & 15 & 14 \\
\hline 1987 & 101 & 53 & 53 & 31 & 31 & 17 & 17 \\
\hline 1988 & 111 & 73 & 66 & 17 & 15 & 21 & 19 \\
\hline 1992 & 93 & 45 & 48 & 31 & 34 & 17 & 18 \\
\hline
\end{tabular}

$\checkmark$ Vimero de lusilidades

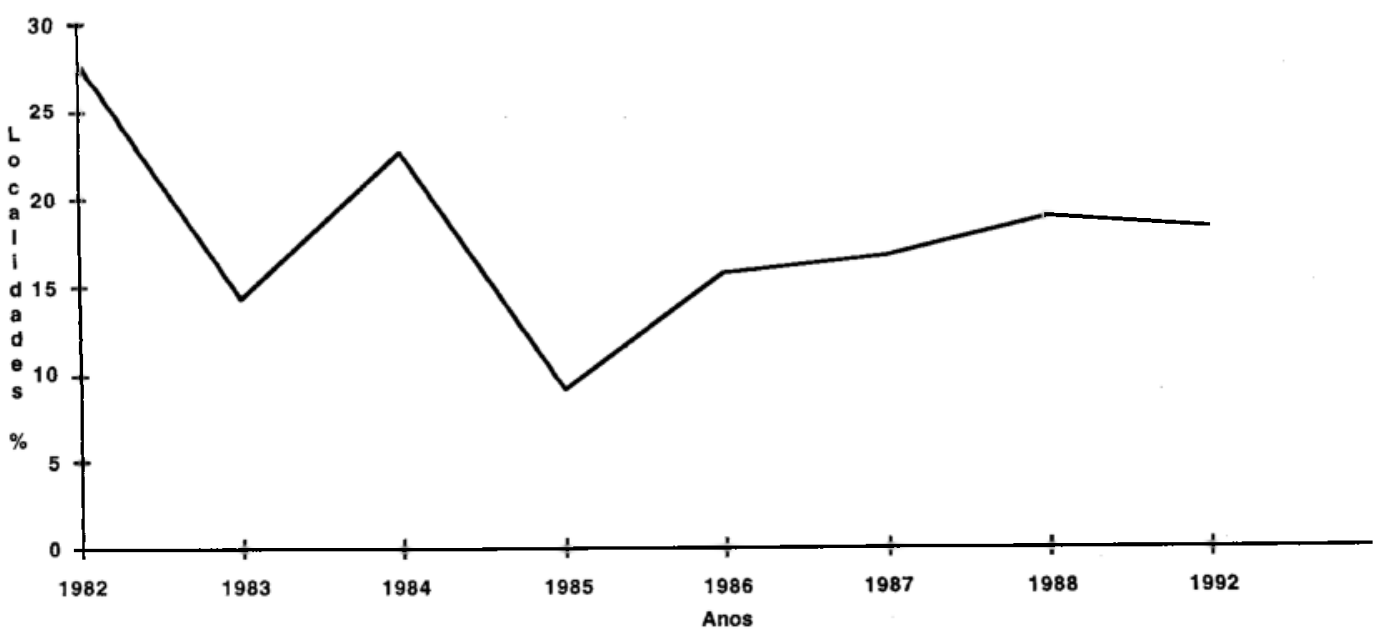

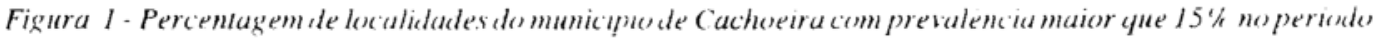
de 1982 a 1992.

tendência é de descenso, cuja magnitude estimada é de $67,9 \%$ no período entre 1982 e 1985 . No ano seguinte, nota-se uma elevação da proporção de aproximadamente $35,7 \%$, a partir do qual, as frequêencias de localidades com prevalências acima de $15 \%$ se mantém estáveis, com variações muito pequenas até o final do período.

A análise da tendência temporal da prevalência de infecção esquistossomótica, conduzida com base em gráticos específicos por cada uma das localidades, revelou distribuições distintas, que foram classificadas em quatro padróes tipicos (Figura 2), denominados de I a IV. Ohserva-se que o Padrão I é de oscilação dos valores da prevalência de modo aproximadamente cíclico. Ou seja, valores elevados estão seguidos por estimativas de prevalências baixas, ciclos que aparentemente, se repetem com uma certa regularidade, aproximadamente por duas vêzes no período. O Padrão II repete, em essência, o traçado do Padrão I: quedas da prevalência seguidas por retorno aos níveis anteriores. Todavia, tal padrão apresenta um traçado mais suave, com curvas em forma de U, e a ocorrência, em sua maioria, de apenas um ciclo completo, ou pelo menos um.menor número de ciclos em comparação ao anterior. Quanto ao Padrão III, este pode ser descrito como de controle da endemia. Inicia-se comaltas prevalências que se reduzem abruptamente, 
Santana VS, Teixeira MG, Santos CCP. Avaliação das ações de controle da infecção esquistossomótica nas localidades de Cachoeira - Bahia, Bacia do Paraguaçu, 1982-1992. Revista da Sociedade Brasileira de Medicina Tropical 29:185-195, mar-abr, 1996.
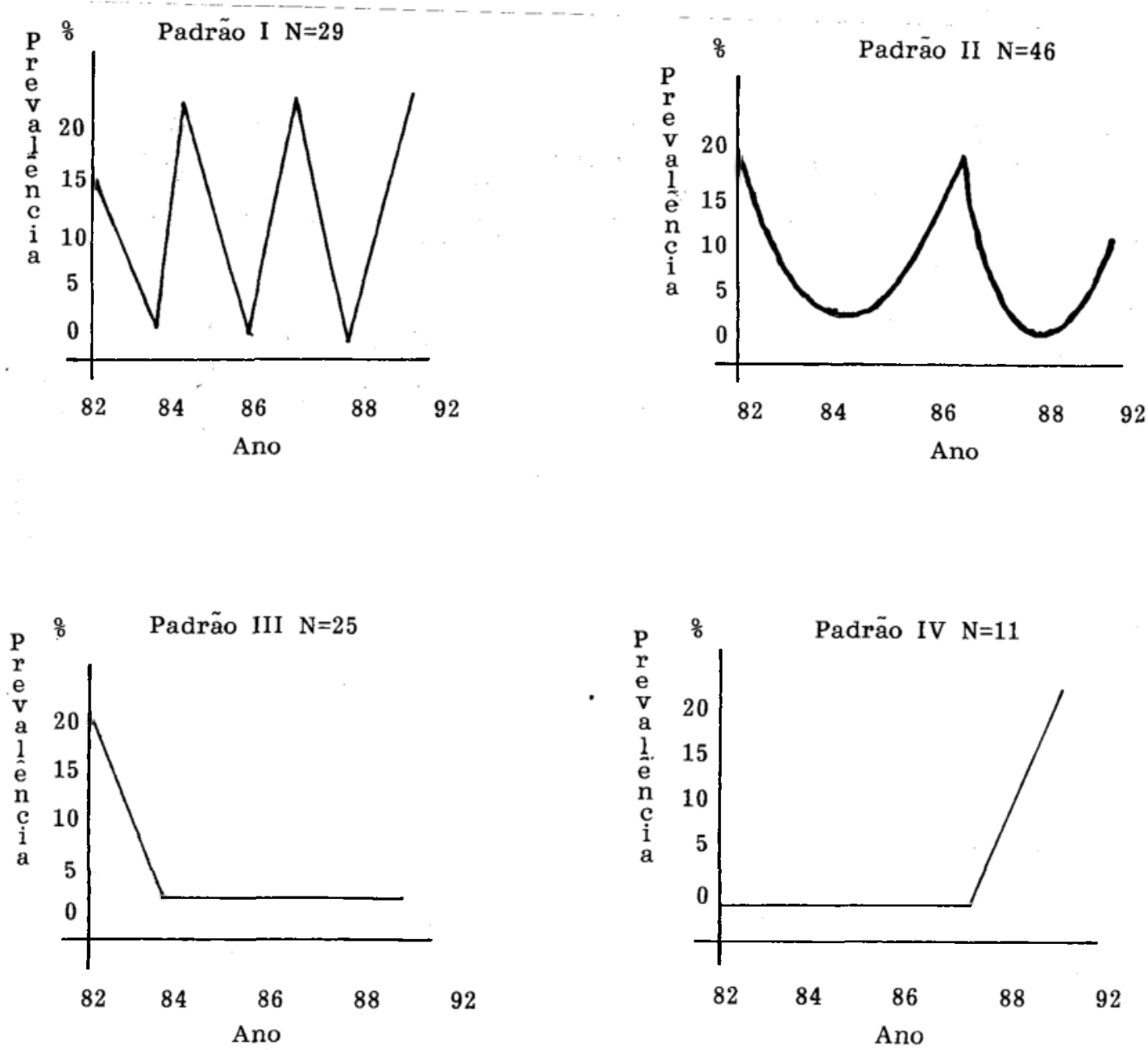

Figura 2 - Padrões típicos de evolução da prevalência de infecção esquistossomótica de localidades do Município de Cachoeira, Bahia. 1982-1992.

mantendo-se no valor zero, ausência de casos, ou muito próximas à este patamar. Ao contrário desse, o Padrão IV evidencia uma elevação, aparentemente geométrica, dos valores de prevalência de infecção esquistossomótica ao longo do período do estudo.

Os dados referentes à distribuição da ocorrência destes padrões podem ser vistos na Tabela 2 . Verifica-se que o padrão mais comum $(41,5 \%)$ é o II, seguido pelo Padrão I com $26,1 \%$ do total das localidades. Conjuntamente, totaliza-se uma ocorrência de $67,6 \%$ desses dois padrões. O Padrão III foi encontradoem 25 localidades que representam $22,5 \%$ do total. O menos freqüente foi o Padrão IV, identificado em apenas 11 dos agregados populacionais $(9,9 \%)$. Note que as médias das populações, estimadas para o ano de 1985 por padrão mostrados na Tabela 2 revelam diferenças que indicam ser as localidades dos padrões de recorrência as mais populosas.

A Figura 3 mostra gráficos do tipo box-plot para as distribuições das prevalências de acordo com os anos do estudo. Verifica-se que todas as curvas encontram-se distorcidas devido a valores

Tabela 2 - Distribuição dos padrões de evolução da prevalência de infecção por $\mathrm{S}$. mansoni nas localidades de Cachoeira, no periodo entre 1982 a 1992.

\begin{tabular}{lcrc}
\hline Padrão & N $^{0}$ & $\%$ & População média em 1985 \\
\hline I & 29 & 26,1 & 586 \\
II & 46 & 41,5 & 279 \\
III & 25 & 22,5 & 181 \\
IV & 11 & 9,9 & 84 \\
\hline Total & 111 & 100,0 & - \\
\hline
\end{tabular}


Santana VS, Teixeira MG, Santos CCP. Avaliação das ações de controle da infeç̧ão esquistossomótica nas localidades de Cachoeira - Bahia, Bacia do Paraguaçu, 1982-1992. Revista da Sociedade Brasileira de Medicina Tropical 29:185-195, mar-abr, 1996.

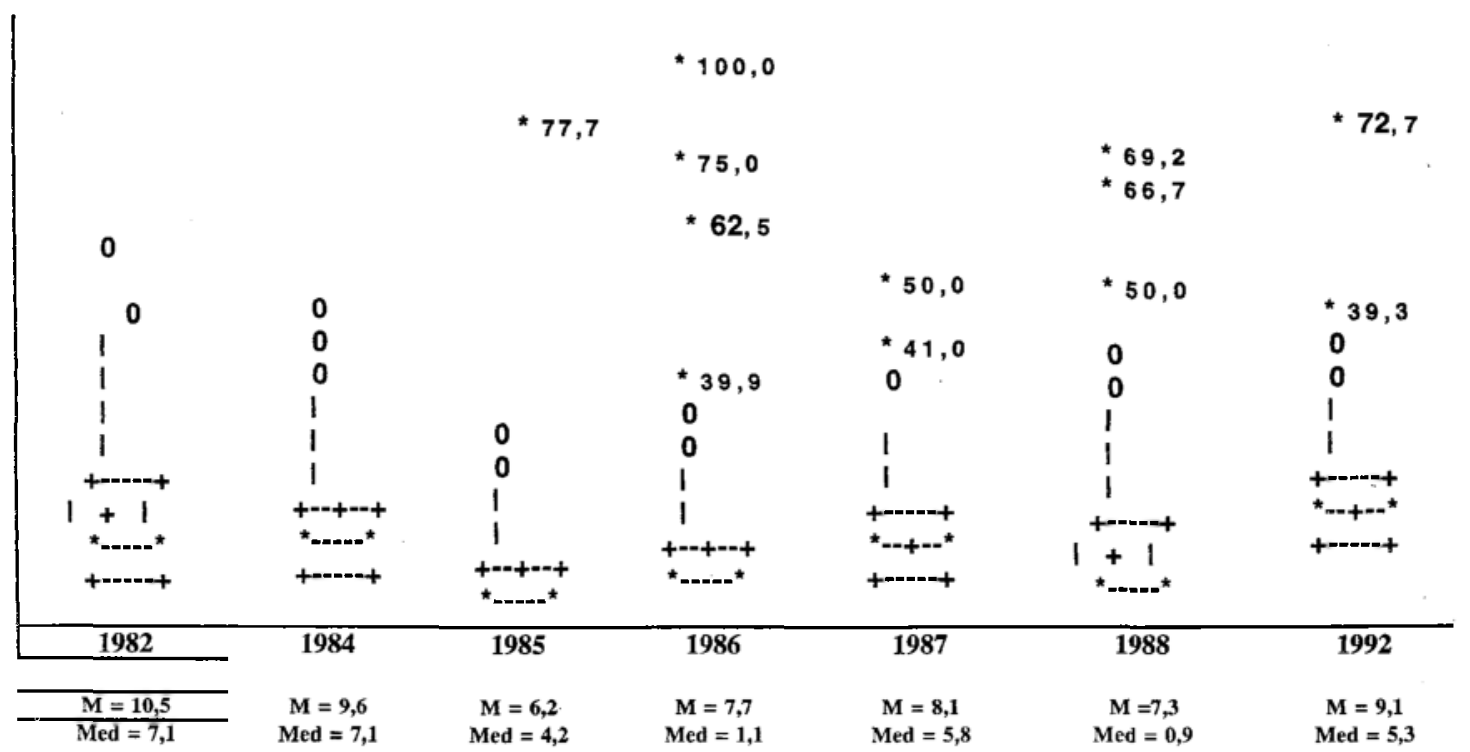

Figura 3 - "Box-plots" das prevalências de injeç̧äo esquistossomótica èm localududes do município de CachoeiraBahia, para cada um dos anos 1982-1992.

Localidades com valores extremos:

1985 - Desterro - 77.7\%;

1986 - Altamira 100\%, Engenho da Praia 75\%, Engenho 62,5\% e Cachoeira E 39,9\%;

1987 - Engenho da Praia 50\%, Campinas B 41\%;

1988 - Pedra Funda 69,2\%, Ponta do Paraguaçu 66,7\%, Rosário 50\%;

1992 - Engenho Novo 72,7\%, Pedra Funda $39,3 \%$

extremos, sempre na direção de altas prevalências. Tais valores não foram tratados como outliers e retirados da análise, porque o objetivo desse estudo é a descrição da ocorrência da prevalência nas situações concretas da realidade dessa investigação. Afastamentos da normalidade ficamevidentes, tanto pelas formas gráficas como também pelas diferenças entre os valores das medianas e médias, mostrados nesta mesma Figura. É de notar que, consistentemente com os dados observados na Figura 2, houve uma redução da média de 10,5 em 1982

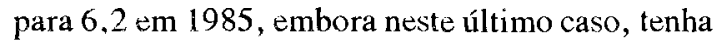
sido verificado também que $50 \%$ das localidades mostravam prevalências de infecção por esquistossomose igual ou menor do que $4,2 \%$, valor abaixo de $5 \%$, definido como indicativo de controle desta endemia. A partir de 1985, as distorções das curvas de prevalências são mais marcadas, devido a ocorrência de valores discrepantes como os de Desterro, Altamira, Engenho da Praia, Engenho Novo, dentre outras localidades (Figura 3).

O registro dos valores extremos na Figura 3 permite uma melhor compreensão desses padrões e sinaliza focos onde o controle epidemiológico não havia sido alcançado, com a infecção em progressão. Todavia, expressão de que as medidas adotadas para o controle produziam efeito sobre a morbidade é o fato dessas localidades, em sua maioria, não se repetirem nos anos seguintes à sua primeira identificação. Assim, embora aparentemente as médias se mantenham com variações muito pequenas, de fato, sua interpretabilidade encontrase comprometida pela não normalidade destas distribuições. Vale ressaltar, a distribuição observada em 1992, quando embora a média esteja próxima à do ano de início do estudo, a mediana em $5,1 \%$ evidencia um significativo impacto do programa, no alcance de valores próximos ao patamar de morbidade desejado.

Por fím, estimou-se a duração média do ciclo de volta dos valores de prevalência aos patamares anteriores para os Padrões I e II. Estes períodos foram estimados em 2,82 anos para o Padrão I e de 3,19 anos para o Padrão II.

Os quatro padrões de evolução de prevalência 
Santana VS, Teixeira MG, Santos CCP. Avaliação das ações de controle da infecção esquistossomótica nas localidades de Cachoeira - Bahia, Bacia do Paraguaçu, 1982-1992. Revista da Sociedade Brasileira de Medicina Tropical 29:185-195, mar-abr, 1996.

foram distribuídos nas localidades sob o mapa do município, não tendo sido observada nenhuma área de concentração geográfica de nenhum deles. Ao contrário, os padrões encontram-se dispersos na área sem qualquer regularidade e muitas vezes encontrou-se padrão de recorrência ao lado de padrão de controle, assim como padrão de expansão ao lado de padrão de controle.

\section{DISCUSSÃO}

Os dados desse estudo revelam que entre 1982 e 1992, nas localidades do município de Cachoeira, Região da Bacia do Paraguaçu, uma área que contou com atividades do programa de controle da esquistossomose da SUCAM, em sua forma padrão para área endêmica, a prevalência de infecção esquistossomótica apresentou uma tendência à redução, principalmente, nos três primeiros anos desse estudo, compreendido entre 1982 e 1985. Verificou-se que tanto havia uma tendência à queda de valores de prevalência quando se considerava as localidades separadamente, como também em termos do conjunto delas, na Região. Neste último caso, os dados revelam que após 1985 , há para o conjunto das localidades um retorno que, felizmente não chega ao nivel de morbidade anterior, permanecendo em um mesmo patamar até o final do período. Isto não se processou de modo linear e homogêneo, mas de acordo com padrões complexos de evolução, que variaram de localidade a localidade, que tentativamente foram classificados em padrões tipo, que se revelaram, em sua maioria, sugestivos de recorrência da prevalência da infecção.

Esta tendência de retorno do comprometimento populacional em relação à infecção por $S$. mansoni, evidencia, entre outros aspectos, a relação entre a eficácia do programa, notadamente, no que se refere aos efeitos esperados da quimioterapia na negativização dos exames pararasitológicos e da efetividade deste programa na sustentação dessa negativização, ou dos seus efeitos sobre a velocidade e ocorrência de reinfecção. Vale ressaltar, que embora os resultados mais importantes decorrentes da quimioterapia, do ponto de vista da saúde pública, sejam a redução da incidência de formas graves e de atenuação do curso da esquistossomose, em sua forma clínica ${ }^{9}$ estes dados não se encontravam disponíveis para a sua análise neste estudo. Assim, o que pode ser inferido destes achados é que, aparentemente, as ações do programa que poderiam se traduzir na sua efetividade, ainda são incipientes, na maioria das localidades estudadas, onde prevaleceu padrões de recorrência da infecção. Ou seja, são limitados os efeitos que podem ser esperados como conseqüência de processos mais complexos de prevenção, como os ambientais, a exemplo da eliminação de caramujos e da circulação de cercárias, da ampliação do acesso da população à água potável ou a equipamentos de lazer ou trabalho que limitem o contato com coleções de água contaminadas. Tem-se ainda os processos de prevenção de alcance individual, onde se destacam as atividades relativas à disseminação de informação sobre o processo de transmissão e práticas adequadas, individuais ou coletivas de prevenção da infecção ou ainda o desenvolvimento de mecanismos que viabilizem a participação da população no conjunto das práticas de saúde. Todos esses processos poderiam alterar o quadro e a evolução da morbidade da infecção esquistossomótica, seja pela redução dos níveis de recorrência, seja pelo aumento de duração do ciclo de recorrência. Infelizmente, nesta etapa preliminar de análise dos dados, não se dispõe ainda de material que possibilite a elucidação dessa questão.

Parece claro que a quimioterapia isoladamente não poderia conduzir à interrupção da transmissão. Portanto, como houve 25 (22\%) localidades onde a prevalência da infecção chegou a zero (Padrão III), é possível hipotetizar que ações preventivas do programa, ou de outra origem, foram efetivas, desde que pudesse ser comprovada a não existência de focos de exposição no ambiente onde viviam essas pessoas. Explanação para este padrão se torna difícil de ser construída, na ausência de inquéritos em profundidade, específicos para estes locais, onde fosse possivel a recuperação do percurso histórico de eventos relacionados com o processo de ocorrência e transmissão da esquistossomose. Ou mesmo da disponibilidade de dados sobre o desenvolvimento de políticas sociais nestas localidades, o que não foi possível de ser identificado, apesar do conhecimento de ter sido esta Região alvo do Programa de Desenvolvimento Regional Integrado do Paraguaçu, o PIDERP. Todavia, permanece ainda a questão: se as ações preventivas foram efetivas, por que isto não se deu uniformemente em todas as localidades desde que 
Santana VS, Teixeira MG, Santos CCP. Avaliação das ações de controle da infecção esquistossomótica nas localidades de Cachoeira - Bahia, Bacia do Paraguaçu, 1982-1992. Revista da Sociedade Brasileira de Medicina Tropical 29:185-195, mar-abr, 1996.

estas atividades são desenvolvidas dentro de um modelo comum? Além desta, pode-se ainda questionar por que em 11 localidades, a tendência foi de aumento exponencial da prevalência (Padrão IV) apesar de estarem sendo tratadas todas as pessoas que apresentaram positividade no exame de fezes, ao lado dos mesmos procedimentos preventivos vinculados ao programa?

Estas indagações fazem sentido no bojo do conhecimento consensual de ser esta parasitose de ocorrência focal. Evidência disto foi a grande amplitude dos valores de prevalência entre as localidades da Região e a não existência de concentração espacial dos quatro padrões de evolução de prevalência. Pode-se também, com os achados desse estudo, afirmar que a evolução é também "focal", ou seja, que os percursos históricos da morbidade se apresentam de modo particular, evidenciando especificidades cuja apreensão somente poderá ser alcançada através do conhecimento de dados complementares àqueles provenientes das coletas rotineiras dos serviços de saúde.

É importante notar que esta análise contemplou não apenas a redução absoluta de magnitudes e formas da evolução da prevalência mas a redução, proporcional, do número de localidades com alta prevalênicia, procedimento ainda incomum nos estudos epidemiológicos sobre avaliação de controle, propiciado com os gráficos box plot. Com estes dados, pode-se concluir que houve redução relativa de localidades com prevalência acima de 5\% enquanto o surgimento de discrepâncias (outliers), ao final do período do estudo, são indicativos de que o fracasso do controle ocorria em situações especiais isoladas mas que não comprometiam o impacto que se refletia no conjunto.

Nesse sentido, as conclusões deste estudo, mesmo que preliminares, são coerentes com os achados de outros autores ${ }^{1432}$ no que diz respeito à redução inicial da prevalência da infecção após quimioterapia. Em todos estes, utilizou-se como base de observação o município. Essa avaliação, por ter se baseado em uma unidade de agregação menor, a localidade, mostra que em parte delas ocorre uma sustentabilidade do impacto epidemiológico. Este achado traduz a distribuição não homogênea da esquistossomose mansônica, como bem registrou Carmo e $\mathrm{col}^{14}$ para o Estado da Bahia como um todo, que se repete dentro das localidades do município de Cachoeira pertencente a uma mesma bacia hidrográfica. Por outro lado, como os padrões considerados de recorrência ocorrem em tempos diferenciados é importante para os programas de controle reverem sua metodologia no que se refere à estratégia de freqüência de retratamento das populações, além de apontar para a necessidade de se identificar quais as ações ou fatores específicos para cada grupo populacional capazes de garantir a continuidade do impacto alcançado. Amaral e col$^{2}$ discutem essa questão, recomendando uma "abordagem alternativa" para localidades que mantêm altas prevalências. A descentralização proposta pelo SUS, pode se constituir em um caminho para essa nova abordagem, em que as diretrizes gerais possam ser discutidas e particularizadas para cada realidade, desde que a transferência de responsabilidade de execução não seja um simples ato burocrático, mas que venha a se constituir em um somatório de esforços de instituições de saúde das três esferas de governo. Por fim, sabendo-se que a quimioterapia influi de forma tão significante na prevenção e evolução das formas graves ${ }^{789}$, os resultados deste estudo podem ser extrapolados no sentido de se concluir que a população desse município foi beneficiada com as ações de controle a que foi submetida.

Os achados dessa avaliação devem ser considerados no contexto da limitação dos estudos ecológicos. Dados de inquéritos transversais seriados, como estes, não tratam da mesma população senso stricto, e de fato a comparabilidade dos dados ano a ano pode ser questionada. Todavia, pode-se pensar como improvável a alteração substancial da composição populacional de pequenas localidades como estas, embora a migração seja ainda significativa nesta região. Desse modo, seria legítima a comparação, dentro dos limites da análise descritiva, que foi utilizada neste trabalho. Vale mencionar o valor dos dados analisados, principalmente no que se refere à duração do intervalo de tempô. Estudos de tendências históricas são, geralmente de difícil viabilização, seja pelos custos como pela dificuldade de manutenção de atividades ao longo do tempo, especialmente, numa realidade onde as descontinuidades dos serviços de saúde são comuns. Estes dados se tornaram disponíveis para a digitação e análise, em parte pela dedicação e extremo profissionalismo dos 
Santana VS, Teixeira MG, Santos CCP. Avaliação das ações de controle da infecção esquistossomótica nas localidades de Cachoeira - Bahia, Bacia do Paraguaçu, 1982-1992. Revista da Sociedade Brasileira de Medicina Tropical 29:185-195, mar-abr, 1996.

funcionários, que mesmo sob precárias condiçoes de trabalho, especialmente, nos anos mais recentes, quando o processo de deterioração das atividades de controle das endemias se tornou notório, mantiveram a coleta e o registro desses dados. Vale ressaltar ainda a determinação de alguns representantes da administração da FNS na avaliação deste programa, que se espera venha a produzir impacto no aperfeiçoamento das ações destinadas ao controle das endemias nesse Estado.

\section{SUMMARY}

This study evaluates the effectiveness of the Schistosomiasis Control Program, PCE, in Cachoeira, state of Bahia-Brazil, in Paraguaçu Basin Area. Data comes from PCE-111 cards of SUCAM/FNS, which are routinety registered in the 111 villages identified during the study follow up, 1982-1992. The S. mansoni infection was diagnosed through Katz-Kato technique for stool exams. Temporal trends of the prevalence of infection were analyzed for each village. Four trend patterns were identified, and the most commom was those of recurrence of infection prevalence (67.6\%). Control of the endemics was achieved in $22.5 \%$ of the villages, while expansion trends were found in only $9.9 \%$ of them. In general, there was found a decrease in the prevalence, particularly, from 1982 to 1985, followed by an increase that do not reach the initial levels. It was observed a relative reduction on the number of villages that shows prevalence below or equal to $5 \%$, that indicates control of the infection. It was not possible to evaluate trends of infection intensity neither the occurrence of severe shistossomiasis disease or related dealhs, that may allow a more complete evaluation of this purstam.

Key-words: Schistosomiasis. Comtrol. Evaltuation. Temperal tends. Espacial distribution.

\section{AGRADECIMENTOS}

Esta pesquisa se tornou possivel pelo trabalho de vários funcionários da FNS, especialmente do Inspetor Sena do Distrito de Pernambués, e do Dr. França da SUCAM/Bahia.

\section{REFERÊNCIAS BIBLIOGRÁFICAS}

1. Abdallah A. Saif M. Koura M. The role of chemoterapy in the control of schistosomiasis: experience in Egypt. Brasília Médica 111:49-54, 1975.

2. Amaral RS. Porto MAS. Evolução e situação atual do controle da esquistossomose no Brasil. Revista da Sociedade Brasileira de Medicina Tropical 27(Supl III):73-90. 1994.

3. Barbosa FS. Control of schistosomiasis: a perspective. Brasilia Médica 111:93-100, 1975.

4. Barreto ML, Carmo EH. Situaçāo de saúde da populaçào brasileira: tendências históricas, determinantes, e impliçaçós para as políticas de saúde. Informe Epidemiológico do SUS III (3/4):7-34, 1994.

5. Benenson AS. El control de las enfermidades transmissibles en el hombre. OPAS, Publ. Cient.. n 538, 1992.

6. Bahia. Centro de Estatística e Planejamento. Informaçóes básicas dos municípios baianos. Recôncavo CEU/CONDER, 1994.

7. Bina JB. Influência da terapêutica específica na evoluçāo da esquistossomose mansoni. Bahia. Tese de Mestrado, Faculdade de Medicina da Universidade Federal da Bahia, Salvador, 1977

8. Bina JC. Prata AR. Regressão da hepatoesplenomegalia pelo tratamento específico da esquistosomosse. Revista da Sociedade Brasileira de Medicina Tropical 16:213-218, 1983.

9. Bina JB. Estudo de variáveis que podem influenciar na evolução da esquistossomose mansônica: efeito da terapêutica específica na interrupção da transmissāo. Bahia. Tese de Doutorado. Faculdade de Medicina da Universidade
Federal da Bahia. Salvador, 1995.

10. Brasil. Conselho de Desenvolvimento Social. Programa especial de controle da esquistossomose no Brasil (PECE) Suplemento I, 1976.

11. Brasil. Ministério da Saúde/Fundação Nacional da Saúde, Controle da esquistossomose: diretrizes técnicas. Brasilia, 1994.

12. Brasil. Ministério da Saúde/Superintendência de Campanhas de Saúde Pública (SUCAM) Normas gerais para o Programa de Controle da Esquistossomose, Brasilia, 1989.

13. Brook RH, Lohr KN. Efficacy, effectiveness, variations, and quality: boundary-crossing research. Medical Care 23:710-722, 1985.

14. Carno EH, Barteto ML. Esquistossomose mansônica no Estado da Bahia. Brasil: tendências históricas e medidas de controle. Cadernos de Saúde Pública 10:425-439, 1994.

15. Coura JR, Argento CA, Conceição MJ, Lewis EM, Santos ML. Magalhāes P. Experiências de campo de oxamniquine oral no tratamento da esquistossomose mansoni. Revista do Instituto de Medicina Tropical de São Paulo 22 (Sup 4):77-84, 1980 .

16. Coura JR, Conceição MJ, Pereira JB. Morbidade da esquistossomose mansoni no Brasil. III - Estudo evolutivo em uma área endêmica no período de 10 anos. Memórias do Instituto Oswaldo Cruz 79:447-453, 1984.

17. Coura JR, Mendonça MZG, Madruga PJ. Tentativa de avaliação do programa éspecial de controle da esquistossomose (PECE) no Estado da Paraíba, Brasil. 
Santana VS, Teixeira MG, Santos CCP. Avaliação das ações de controle da infecção esquistossomótica nas localidades de Cachoeira - Bahia, Bacia do Paraguaçu, 1982-1992. Revista da Sociedade Brasileira de Medicina Tropical 29:185-195, mar-abr, 1996.

Revista da Sociedade Brasileira de Medicina Tropical 20:67-76, 1987.

18. CNPq. Epidemiologia e controle de doenças endêmicas do Nordeste Semi Árido. (Mineo), 1978.

19. Freitas CA. Situação atual da esquistossomose no Brasil. Rev. Bras. de Malariologia e Doenças Tropicais 3-19, s.d.

20. Jordan P. Schistosomiasis-research to control. The American Journal of Tropical Medicine and Hygiene 26:877-886, 1977.

21. Lima e Costa MFF, Rocha RS, Coura Filho P, Katz NA. A 13-year follow-up of treatment and snail control in an area endemic for Schistosoma Mansoni in Brazil: incidence od infection and reinfection. Bulletin of the World Health. Organization $71: 197-205,1993$.

22. Lipsey MW. Theory as method: small theories of treatments. In: Sechrest L, Perrin E, Bunker J (eds) Research methodology: strengthening causal interpretations of nonexperimental data. Conference Procedings. U.S. Department of Health and Human Services, (May), 1990.

23. Organización Mundial de la Salud. Evalución de los programas de salud: normas fundamentales para su aplicación en el proceso de gestión para el desarrollo nacional de la salud. Ginebra, 1981.

24. Prata A, Bina JC, Barreto AC, Alecrim MG. Attempt to control the schistosomiasis transmission by oxamniquine in an hyperendemic locality. Revista do Instituto de Medicina Tropical de São Paulo 22(Sup 4):65-72, 1980.

25. Pellon A, Teixeira I. Inquérito helmintológico escolar em cinco estados das regiōes Leste, Sul e Centro Oeste. Ministério da Educação e Saúde, Departamento Nacional de Saúde, Divisão de Organização Sanitária. Apresentado no XI Congresso Brasileiro de Higiene, Curitiba, 1953.

26. SAS. Software Institute, version 6, First Edition. SAS Institute Inc., Cary, NC, USA, 1990.

27. Santos ML, Coura R. Morbidade da esquistossomose no Brasil. IV - Evolução em pacientes tratados e seus controles. Memórias do Instituto Oswaldo Cruz 81:53-60, 1986.

28. Santos ML. Esquistossomose mansoni: estudo da morbidade e interferência da terapêutica específica em uma área endêmica. Tese de Mestrado. Faculdade de Medicina da Universidade Federal do Rio de Janeiro, Rio de Janeiro, 1978.

29. Silva JR. Valor e importância do tratamento específico da esquistossomose mansoni no campo da profilaxia. Revista Brasileira de Medicina 14:524-526, 1957.

30. Silva LV, Formigli VLA. Avaliação em Saúde: limites e perspectivas. Cadernos de Saúde Pública 10:80-91, 1994.

31. Silveira AC. Controle da Esquistossomose no Brasil. Memórias do Instituto Oswaldo Cruz 84(Sup 1):91-104, 1989.

32. Sleigh AC, Mott KE, França Silva JTF, Muniz TM, Mota EA, Barreto ML, Hoff R, Maguire JH, Lehman JS, Sherlock IA. A three year follow-up of chemotherapy with oxamniquine in a Brasilian community with endemic shistosomiasis mansoni. Transactions of The Royal Society of Tropical Medicine and Hygiene 72:234-238, 1981.

33. Zein AZ. Spontaneous reduction in Schistosoma mansoni infection in endemic communities of lake Tana basin, north-western Ethiopia. Transactions of The Royal Society of Tropical Medicine and Hygiene 83:656-658, 1989. 